\title{
Spontaneous Low Frequency Oscillations in Acute Ischemic Stroke - A Near Infrared Spectroscopy (NIRS) Study
}

Dorte Phillip ${ }^{1}$, Henrik W Schytz ${ }^{1}$, Helle K Iversen ${ }^{2}$, Juliette Selb ${ }^{3}$, David A Boas ${ }^{3}$ and Messoud Ashina ${ }^{{ }^{*}}$

${ }^{1}$ Department of Neurology, University of Copenhagen, Denmark

${ }^{2}$ Stroke Unit, Department of Neurology, University of Copenhagen, Denmark

${ }^{3}$ The Optics Division, Harvard Medical School, USA

*Corresponding author: Messoud Ashina, Danish Headache Center, Glostrup Hospital, Ndr Ringvej 69, DK-2600 Glostrup Denmark, Tel: +45 38633054 ; Fax: +45 38633839; E-mail: Ashina@dadlnet.dk

Received date: Aug 31, 2014, Accepted date: Oct 27, 2014, Published date: Oct 31, 2014

Copyright: (C) 2014 Phillip D, et al. This is an open-access article distributed under the terms of the Creative Commons Attribution License, which permits unrestricted use, distribution, and reproduction in any medium, provided the original author and source are credited.

\begin{abstract}
Background and purpose: Continuous wave near infrared spectroscopy (NIRS) is a non-invasive bed-side optical method to detect changes in oxygenated $(\mathrm{oxyHb})$ and deoxygenated hemoglobin (deoxy $\mathrm{Hb})$ in the outermost layers of the cerebral cortex. Cortical oxyHb low frequency oscillations (LFOs) in the $0.09-0.11 \mathrm{~Hz}$ range are affected by changes in cerebral autoregulation (CA), which is altered following stroke. We examined oxyHb LFOs at bed-side as a marker of $\mathrm{CA}$ in the subacute phase in stroke patients with or without recombinant tissue plasminogen activator thrombolytic therapy.
\end{abstract}

Methods: We recruited 29 patients admitted to the stroke unit with symptoms of ischemic stroke. 11/29 patients received thrombolytic therapy. NIRS examination was conducted 2 days (median time) from stroke onset. NIRS optodes were placed on each side of the head with a $3 \mathrm{~cm}$ source-detector distance. Using transfer function analysis, inter-hemispheric phase shift and amplitude ratio of the oxyHb oscillations in the $0.09-0.11 \mathrm{~Hz}$ range were assessed.

Results: The correlation between NIHSS scores at admission and oxyHb parameters revealed a significant positive correlation between stroke severity at admission and inter-hemispheric phase shift $(\mathrm{P}=0.028)$. The oxyHb absolute inter-hemispheric phase shift was significantly less in patients receiving thrombolytic therapy compared to non-thrombolytic therapy patients $\left(3^{\circ}\right.$ vs. $\left.23^{\circ}, P=0.005\right)$.

Conclusions: Stroke severity correlates with the degree of impaired cortical CA and stroke patients receiving thrombolytic therapy might have less severely impaired CA. NIRS detects alteration in cortical oxyHb LFOs between hemispheres in stroke patients in the subacute phase and may be a feasible method to explore changes at bed-side in a stroke unit.

Keywords: Cerebral autoregulation; Low frequency oscillations; Stroke; Near infrared spectroscopy; Thrombolytic treatment; rtPA thrombolytic therapy; Transfer function analysis

\section{Introduction}

Low frequency oscillations (LFOs), known as Mayer waves [1], at $0.1 \mathrm{~Hz}$ of systemic and cerebral vessels are affected by neuronal [2], metabolic [3] and myogenic [4] stimuli. Spontaneous LFOs are relatively easy to investigate under clinical conditions, as they only require the patient to lie still and breathe normally during examination $[5,6]$. In cerebral vessels, LFOs are affected by changes in cerebral autoregulation (CA) [6] and have primarily been investigated by detecting the relationship between the systemic arterial blood pressure $(\mathrm{ABP})$ and flow velocity changes in the middle cerebral artery (Vmca) using transcranial Doppler (TCD) $[7,8]$.

In ischemic stroke cortical vessels may be affected, and it is therefore of interest to directly measure local changes in cortical resistance vessels. Studies of LFOs using TCD have shown changes in CA following ischemic stroke, which are dependent on the time interval after ictus $[9,10]$ and infarct location [11]. Changes in CA are associated with the clinical outcome following ischemic stroke $[10,12]$.

Near infrared spectroscopy (NIRS) is a non-invasive optical bedside method to investigate regional changes in oxygenated (oxyHb) and deoxygenated haemoglobin (deoxyHb) in the outermost layers of the cerebral cortex [13]. Specific cortical oxyHb LFOs can be altered by brain activity [14-16], metabolic [5] and sympathetic changes [17]. Furthermore, phase shift and gain between ABP and oxyHb LFOs may correlate to Vmca changes in carotid artery disease [18]. Subject variation in anatomy [19] may challenge inter-subject comparisons using NIRS. By measuring oxyHb inter-hemispheric phase shift, amplitude ratio and inter-hemispheric cross correlation (IHCC) $[19,20]$, these issues are minimized, as each subject serves as their own control. Thus, NIRS may be an ideal tool to investigate subacute cortical changes in CA in stroke patients at bedside.

In the present study we aimed to explore changes in spontaneous cortical oxyHb LFOs measured with NIRS following ischemic stroke. We hypothesized that oxyHb LFOs would reveal differences between the symptomatic and contralateral side for ABP-oxyHb phase shift and gain as well as oxyHb amplitude. We expected NIH stroke scale 
Citation: Phillip D, Schytz HW, Iversen HK, Selb J, Boas DA, et al. (2014) Spontaneous Low Frequency Oscillations in Acute Ischemic Stroke A Near Infrared Spectroscopy (NIRS) Study. J Neurol Neurophysiol 5: 241. doi:10.4172/2155-9562.1000241

Page 2 of 5

(NIHSS) scores at admission and $24 \mathrm{~h}$ after to be correlated with ABPoxyHb and oxyHb parameters. Furthermore, we examined ABPoxyHb phase shift and gain, oxyHb amplitude as well as interhemispheric oxyHb parameters for differences between thrombolytic and non-thrombolytic therapy patients.

\section{Material and Methods}

We recruited 29 patient (15 male, mean age 67, range 39-91) admitted to the Stroke Unit, Glostrup Hospital in Denmark from
September 2009 to September 2011 with symptoms of stroke in the territory of the internal carotid artery (Table 1). 11 out of 29 patients received rtPA thrombolytic therapy before participating in the study. The median time of examination was day 2 (range 1-5) after infarct onset. The Biomedical Research Ethics committee in the Capital Region of Denmark approved the study (H-B-2008-088). All subjects gave informed consent to participate in the study, which was undertaken in accordance with the Helsinki Declaration of 1964, as revised in Edinburgh in 2000.

\begin{tabular}{|c|c|c|c|}
\hline & $\begin{array}{l}\text { Thrombolytic } \\
\text { therapy } \\
(n=11)\end{array}$ & $\begin{array}{l}\text { No thrombolytic } \\
\text { therapy } \\
(n=18)\end{array}$ & $P$ value \\
\hline Age & $65 \pm 16$ & $68 \pm 12$ & 0.653 \\
\hline Male & 8 & 7 & 0.648 \\
\hline Hypertension & 7 & 10 & 0.180 \\
\hline Diabetes & 1 & 0 & \\
\hline Previous stroke & 3 & 3 & 0.227 \\
\hline Hypercholesterolemia & 5 & 4 & 0.754 \\
\hline NIHSS at admission & $5(2-11)$ & $2.5(0-14)$ & 0.025 \\
\hline Time of recording & $1(0-2)$ & $2(0-4)$ & 0.06 \\
\hline Hemisphere affected, left & 7 & 9 & 0.481 \\
\hline Remission after $24 \mathrm{H}$ & 4 & 5 & 0.774 \\
\hline Systolic blood pressure mmHg & $122 \pm 18$ & $144 \pm 29$ & 0.031 \\
\hline Diastolic blood pressure $\mathrm{mmHg}$ & $77 \pm 13$ & $82 \pm 12$ & 0.290 \\
\hline \multicolumn{4}{|l|}{ Smoking: } \\
\hline Yes & 3 & 4 & \\
\hline No & 5 & 10 & \\
\hline Previous & 3 & 4 & \\
\hline
\end{tabular}

Table 1: Baseline clinical characteristics of patients

\section{Near infrared spectroscopy}

We used continuous wave NIRS (NIRS2; TechEn Inc, Milford, MA, USA) at a sampling rate of $200 \mathrm{~Hz}$. The NIRS optodes were placed with 1 source and 1 detector on each side of the head with a distance of $3 \mathrm{~cm}$ between source and detector, each light source emitting two different wavelengths $(690 \mathrm{~nm}$ and $830 \mathrm{~nm})$. On each hemisphere the source was placed on the forehead, carefully avoiding the midline sinus, and with the detector placed laterally.

\section{Arterial blood pressure}

Continuous non-invasive $\mathrm{ABP}$ recording was collected via a finger plethysmograph (CNAP500, CNSystems, Graz, Austria) using the left hand, positioned at heart level. Data from the finger plethysmograph was stored with the NIRS data for subsequent off-line analysis.

\section{Procedures}

Before the experiments, each participant underwent a general physical and neurological examination. All experiments were performed with the patients placed in a supine position in a silent room with constant temperature and the light dimmed. After $15 \mathrm{~min}$ of rest in the supine position, data acquisition was started, with a 10 min trial of spontaneous breathing.

\section{Data analysis}

To remove motion artefact, we used spline interpolation as previous described [21]. In short, periods of motion artefacts are defined, and each period is modulated using spline interpolation. The modelled data is then subtracted from that period of original data.

All signals were analysed in successive $50 \%$ overlapping time segments of 100 seconds. The NIRS light intensities at $690 \mathrm{~nm}$ and 830 
$\mathrm{nm}$ were first converted to time series of variations in oxyHb concentrations using the modified Beer-Lambert law.

On each time segment, we then computed the power spectra of all signals (ABP, oxyHb), the coherence spectra for ABP-oxyHb and the complex transfer function for ABP-oxyHb (The Math Works, Inc, Natick, MA, USA). The phase shift and gain between ABP oscillations and oxyHb were obtained as the phase and absolute value of the complex transfer function respectively. The LFO frequency was selected as the frequency with maximal $\mathrm{ABP}$ oscillation power in the $0.09-0.11 \mathrm{~Hz}$ range. In this manner, for each run we obtained 11 (10 min run) overlapping segments each characterized by a coherence value, a phase shift, and a gain for ABP-oxyHb. We selected the segments for which the coherence was above an arbitrary threshold of 0.7 , and averaged the gain (linear average) and the phase shift (circular average) over these selected segments. If the total number of segments with coherence above 0.7 was less than 3 for a specific run, we discarded that run. The oxyHb inter-hemispheric phase shift was defined as the phase shift between the stroke side and contralateral sides. Thus, a positive phase shift implied that the stroke side was ahead in time compared to the contralateral side. The oxyHb interhemispheric amplitude ratio was defined as the amplitude ratio of contralateral/stroke sides. Thus, an amplitude ratio above 1 implied that the amplitude was lower on the stroke side compared to the contralateral side. Inter-hemispheric cross correlation (IHCC) was defined as the cross correlation of changes in the optical density at 830 $\mathrm{nm}$. An IHCC of 1 indicates that the hemispheres are in phase (phase delay 0 ), and an IHCC of -1 indicates a $180^{\circ}$ phase delay.

We also analysed the absolute values of oxyHb inter-hemispheric phase shift, and the oxyHb amplitude ratio of the hemisphere with higher amplitude over that of lower amplitude (ratio above 1, referred to below as the "absolute oxyHb amplitude ratio") This allows us to investigate the inter-hemispheric synchronicity and amplitude difference independently of whether the infarct side is systematically behind or ahead in time, or whether it presents larger or smaller amplitude of oscillations than the contralateral side.

All NIRS data was assessed blindly in respect to symptomatic and non-symptomatic side.

\section{Statistics}

Data are presented as mean values and standard deviation $( \pm S D)$.

The primary endpoint was: 1) To compare the infarct versus the non-infarct side for $\mathrm{ABP}-\mathrm{OxyHb}$ phase and gain as well as $\mathrm{OxyHb}$ amplitude.

The secondary end points were: 1) The correlation between NIHSS score at admission to $\mathrm{ABP}-\mathrm{oxyHb}$ phase shift, gain and oxyHb amplitude on the symptomatic side, and oxyHb inter-hemispheric phase shift, amplitude ratio and IHCC.

2) To compare thrombolytic therapy patients vs. non-thrombolytic therapy patients on the symptomatic side for ABP-oxyHb phase shift and gain as well as oxyHb amplitude, and oxyHb inter-hemispheric phase shift, amplitude ratio and IHCC.

3) The correlation between NIHHS score changes from admission to $2 \mathrm{~h}$ and $24 \mathrm{~h}$ to $\mathrm{ABP}$-oxyHb phase shift and gain and oxyHb amplitude on the symptomatic side, and oxyHb inter-hemispheric phase shift, amplitude ratio and IHCC.
In the group of patients not receiving thrombolytic treatment we made a post hoc analysis comparing patients with manifest infarction versus transient ischemic attack (TIA) patients for $\mathrm{ABP}-\mathrm{oxyHb}$ phase shift and gain as well as oxyHb amplitude on the symptomatic side, and oxyHb inter-hemispheric phase shift, amplitude ratio and IHCC.

To test the differences between the infarct and non-infarct hemisphere for amplitude, we used Wilcoxon's Signed Ranks test. Differences between thrombolytic therapy patients and nonthrombolytic therapy patients were tested using Mann-Whitney test and McNemar test for dichotomic data. The relationship between NIHSS score and NIRS variables were tested by Spearman's correlation, as well as the relationship between changes in NIHSS and NIRS parameters. All analyses were performed with IBM SPSS (20.0). We made no adjustment for multiple analyses. Thus, the level of significance at 0.05 was accepted for each comparison.

\section{Results}

All 29 patients completed the study and clinical characteristics of the patients are shown in Table $1.4 / 11$ subjects in the thrombolytic group and 6/18 in the non-thrombolytic group received inhibitors of platelet aggregation prior to the admission. The coherence in ABPoxyHb phase shift and gain was too low, and was therefore not analysed further. For oxyHb inter-hemispheric amplitude ratio and phase shift we excluded $10 \%$ of the runs due to low coherence.

The oxyHb oscillation amplitude on the infarct side was not different compared to the contralateral side $(0.014 \pm 0.030$ vs. $0.017 \pm$ $0.032, \mathrm{P}=0.758$ ). The oxyHb absolute inter-hemispheric phase shift was significantly smaller in patients receiving thrombolytic therapy compared to non-thrombolytic therapy patients $\left(3^{\circ}\right.$ vs. $\left.23^{\circ}, \mathrm{P}=0.005\right)$. For the other oxyHb variables we found no differences between patient groups (Table 2).

\begin{tabular}{|l|l|l|l|}
\hline & $\begin{array}{l}\text { Thrombolytic } \\
\text { therapy }(\mathbf{n}=11)\end{array}$ & $\begin{array}{l}\text { No thrombolytic } \\
\text { therapy }(\mathbf{n}=18)\end{array}$ & $\begin{array}{l}\text { P- } \\
\text { value }\end{array}$ \\
\hline Amplitude on stroke side & $0.029( \pm 0.046)$ & $0.005( \pm 0.005)$ & 0.170 \\
\hline Inter-hemispheric phase shift & $-1^{\circ}\left( \pm 5^{\circ}\right)$ & $-13^{\circ}\left( \pm 48^{\circ}\right)$ & 0.550 \\
\hline $\begin{array}{l}\text { Absolute inter-hemispheric } \\
\text { phase shift }\end{array}$ & $3^{\circ}\left( \pm 3^{\circ}\right)$ & $23^{\circ}\left( \pm 44^{\circ}\right)$ & 0.005 \\
\hline $\begin{array}{l}\text { Inter-hemispheric amplitude } \\
\text { ratio }\end{array}$ & $0.990( \pm 0.312)$ & $1.519( \pm 1.161)$ & 0.186 \\
\hline $\begin{array}{l}\text { Absolute inter-hemispheric } \\
\text { amplitude ratio }\end{array}$ & $1.304( \pm 0.313)$ & $1.773( \pm 1.038)$ & 0.073 \\
\hline $\begin{array}{l}\text { Inter-hemispheric cross } \\
\text { correlation }\end{array}$ & $0.6\left( \pm 0.2^{\circ}\right)$ & $0.5( \pm 0.3)$ & 0.500 \\
\hline
\end{tabular}

Table 2: Differences in low frequency oscillation variables between patients with or without thrombolytic therapy

The correlation between NIHSS scores at admission and oxyHb parameters revealed a significant positive correlation between stroke severity at admission and inter-hemispheric phase shift ( $\mathrm{r} 0.430$, $\mathrm{P}=0.028$, Figure 1 ). For the other oxyHb variables no correlations were found (Table 3). Post hoc analysis revealed no correlation between systolic ABP and absolute inter-hemispheric phase $(\mathrm{p}=0.755)$ or NIHSS at admission $(\mathrm{p}=0.452)$. 
Page 4 of 5

\begin{tabular}{|l|l|l|}
\hline & $\begin{array}{l}\text { Correlation coefficient } \\
\text { Spearman's rho }\end{array}$ & P-value \\
\hline Amplitude on stroke side & 0.154 & 0.426 \\
\hline Inter-hemispheric phase shift & 0.430 & 0.028 \\
\hline $\begin{array}{l}\text { Absolute inter-hemispheric phase } \\
\text { shift }\end{array}$ & -0.099 & 0.631 \\
\hline Inter-hemispheric amplitude ratio & 0.062 & 0.762 \\
\hline $\begin{array}{l}\text { Absolute inter-hemispheric } \\
\text { amplitude ratio }\end{array}$ & -0.017 & 0.933 \\
\hline Inter-hemispheric cross correlation & -0.165 & 0.393 \\
\hline
\end{tabular}

Table 3: Correlation between NIH stroke scale (NIHSS) values at admission and low frequency oscillation variables. $P$ values: Spearman's correlation.

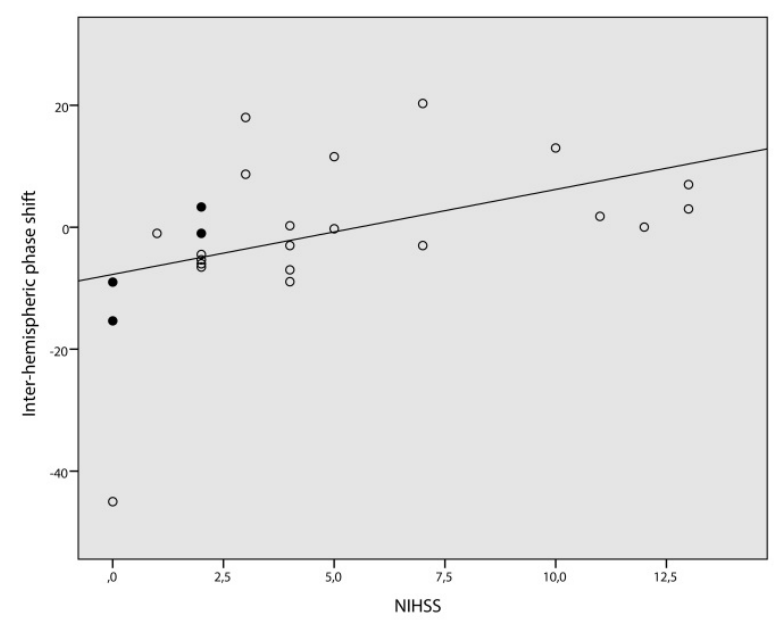

Figure 1: Correlation between NIHSS at admission and interhemispheric phase shift. Full markers are patients with transient ischemic attack (TIA). One subject with infarction on CT scan had a NIHSS of 0 and presented with cognitive symptoms.

In patients receiving thrombolytic treatment, the changes in NIHSS between admission and 2 and $24 \mathrm{~h}$ revealed no correlation for any oxyHb variables.

Post hoc analysis of the 13 patients with manifest infarction versus 5 patients with TIA revealed no statistical differences (Table 4).

\begin{tabular}{|l|l|l|l|}
\hline Channel & $\begin{array}{l}\text { Stroke } \\
(\mathbf{n}=13)\end{array}$ & $\begin{array}{l}\text { TIA } \\
(\mathbf{n}=5)\end{array}$ & $\begin{array}{l}\text { P- } \\
\text { values }\end{array}$ \\
\hline Amplitude & $\begin{array}{l}0.006 \quad(\quad \pm \\
0.006)\end{array}$ & $\begin{array}{l}0.004 \\
0.003)\end{array}$ & $(\quad \pm 0.843$ \\
\hline Inter-hemispheric phase shift & $-15^{\circ}\left( \pm 57^{\circ}\right)$ & $-8^{\circ}\left( \pm 6^{\circ}\right)$ & 0.267 \\
\hline $\begin{array}{l}\text { Absolute inter-hemispheric phase } \\
\text { shift }\end{array}$ & $28^{\circ}\left( \pm 51^{\circ}\right)$ & $8^{\circ}\left( \pm 6^{\circ}\right)$ & 0.327 \\
\hline Inter-hemispheric amplitude ratio & $\begin{array}{l}1.597 \quad(\quad \pm \\
1.331)\end{array}$ & $\begin{array}{l}1.303 \\
0.549)\end{array}$ & $( \pm \pm$ \\
\hline
\end{tabular}

\begin{tabular}{|c|c|c|c|}
\hline $\begin{array}{l}\text { Absolute inter-hemispheric amplitude } \\
\text { ratio }\end{array}$ & $\begin{array}{l}1.891 \\
1.184)\end{array}( \pm$ & $\begin{array}{lll}1.448 & (\quad \pm \\
0.401) & \end{array}$ & 0.602 \\
\hline Inter-hemispheric cross correlation & $0.5( \pm 0.3)$ & $0.7( \pm 0.2)$ & 0.218 \\
\hline
\end{tabular}

Table 4: Differences in low frequency oscillation variables for nonthrombolytic patients with transient ischemic attack (TIA) versus manifest infarction. P-values: Mann-Whitney test.

\section{Discussion}

The major outcome of this study was a positive correlation between NIHSS scores at admission and the oxyHb inter-hemispheric phase shift. Furthermore, we showed that oxyHb absolute inter-hemispheric phase shift was significantly smaller in ischemic stroke patients receiving thrombolytic therapy compared to non-thrombolytic therapy patients. For the remaining oxyHb variables no differences were found between hemispheres, therapy groups and NIHSS score.

Recently, Obrig [22] suggested stroke and cerebrovascular disease as a promising area for NIRS in clinical neurology and that subacute stroke might be the most relevant field that NIRS could reach the clinical neurologist. The present study is, to our knowledge, the first to analyse LFOs with NIRS following ischemic stroke in the subacute phase. To date, no studies are available to directly compare our results to, and no gold standard is provided on how to investigate stroke patients with NIRS. We used a variety of measures to investigate possible changes in $\mathrm{CA}$ and found that $\mathrm{ABP}-\mathrm{oxyHb}$ had too low coherence to obtain valid data. Whether this finding is due to motion artefacts or pathological loss of coherence is unknown. Furthermore, we found no differences in oxyHb amplitudes, which might be due to arterioles distal to the infarct area dilated compensatively to ensure oxygen delivery. However, this effect might have been attenuated due to LFOs decreasing with age [23] and cerebral microangiopathy [24]. Of the 29 patients recruited, only 7 patients had clear involvement of cerebral cortex based upon symptoms and imaging at admission, and two of these were without any neurological signs after $24 \mathrm{~h}$ (both received thrombolytic therapy). The lack of difference between hemispheres may be due to impaired CA in both hemispheres, which has previously been shown in patients with lacunar strokes assessed by ABP-Vmca LFOs [11].

Using NIRS, a previous pilot study (20) examined 9 patients with ischemic stroke and analysed IHCC within cardiac $(0.7-3 \mathrm{~Hz})$ and respiratory $(0.15-0.7 \mathrm{~Hz})$ frequency spectrums. This study showed a smaller IHCC in stroke compared to healthy controls. This suggests a de-synchronization between hemispheres following ischemic stroke, but the change may also be caused by $44 \%$ of the patients in the study having a high-degree ipsilateral carotid disease. In the present study, we found a significantly smaller oxyHb absolute inter-hemispheric phase shift in patients treated with thrombolytic therapy compared to patients not receiving thrombolytic therapy. This reflects desynchronization in the non-thrombolytic group and possible a decline in CA. The patients undergoing thrombolytic therapy were examined after administration of thrombolytic treatment and they had in general a good outcome (full remission in $36 \%$ vs. $28 \%$ ) (Table 1 ). It is therefore possible, that the treatment had an effect on the variability and CA as well as on the NIHSS. In support, a previous study has measured LFOs ABP-Vmca correlation coefficient and phase in patients undergoing successful or unsuccessful rtPA thrombolytic therapy [10]. The study showed that CA was increasingly impaired, mainly on the affected side, over the first 5 days of major ischemic 
stroke after unsuccessful rtPA thrombolysis, but was bilaterally preserved in minor stroke after successful rtPA thrombolysis, indicating no separate detrimental effect of rtPA on the cerebral autoregulatory mechanism. Collectively, these studies suggest that disturbance in CA following ischemic stroke is not caused by the rtPA thrombolytic therapy, but by the degree of stroke severity.

There are some limitations in the present study. We did not examine healthy controls and it is possible that the inter-hemispheric variables would be different between patients and controls. The patients undergoing thrombolytic treatment were investigated earlier than the rest, and it is possible that CA alterations changes over time, which may blur the results. Furthermore, the patients present a mixture of lacunar and cortical infarction, with 13 patients without clear evidence of infarct localization and only 7 patients had clear involvement of cerebral cortex based upon imaging and symptoms. This may lead to false negative results. In addition, we did not correct for the contribution of skin vasculature, which may bias our results. This is a small explorative study with a low number of subjects, and we did not adjust for multiple comparisons in statistical testing. Therefore the results need to be handled with caution and further investigations are needed.

In conclusion, our results suggest that NIRS may detect asymmetry in cortical LFOs between hemispheres in stroke patients in the subacute phase. The study shows that NIHSS score at admission is correlated to the LFOs oxyHb inter-hemispheric phase shift. Patients receiving thrombolytic therapy seem to have less impaired $\mathrm{CA}$, which can be measured using the absolute inter-hemispheric phase shift. Thus, NIRS may be a feasible method to explore changes in ischemic stroke patients using inter-hemispheric phase shift analysis. Further studies are needed to prospectively investigate changes in cortical LFOs using NIRS in cerebrovascular disease.

\section{Acknowledgements}

The study was supported by the Lundbeck Foundation via the Lundbeck Foundation Center for Neurovascular Signaling (LUCENS), the University of Copenhagen, the Augustinus Foundation and the Toyota Foundation.

\section{References}

1. Mayer S (1876) Studien zur physiologie des herzens und der blutgefasse. sitz kaiser akad wiss 74, 281-307.

2. Mayhew JE, Askew S, Zheng Y, Porrill J, Westby GW, et al. (1996) Cerebral vasomotion: a $0.1-\mathrm{Hz}$ oscillation in reflected light imaging of neural activity. Neuroimage 4: 183-193.

3. Hudetz AG, Smith JJ, Lee JG, Bosnjak ZJ, Kampine JP (1995) Modification of cerebral laser-Doppler flow oscillations by halothane, PCO2, and nitric oxide synthase blockade. Am J Physiol 269: H114-120.

4. Auer LM, Gallhofer B (1981) Rhythmic activity of cat pial vessels in vivo. Eur Neurol 20: 448-468.

5. Obrig H, Neufang M, Wenzel R, Kohl M, Steinbrink J, et al. (2000) Spontaneous low frequency oscillations of cerebral hemodynamics and metabolism in human adults. Neuroimage 12: 623-639.

6. Schytz HW, Hansson A, Phillip D, Selb J, Boas DA, et al. (2010) Spontaneous low-frequency oscillations in cerebral vessels: applications in carotid artery disease and ischemic stroke. J Stroke Cerebrovasc Dis 19: 465-474.

7. Reinhard M, Muller T, Guschlbauer B, Timmer J, Hetzel A (2003). Transfer function analysis for clinical evaluation of dynamic cerebral autoregulation--a comparison between spontaneous and respiratoryinduced oscillations. Physiol Meas 24: 27-43.

8. Aries MJ, Elting JW, De Keyser J, Kremer BP, Vroomen PC (2010) Cerebral autoregulation in stroke: a review of transcranial Doppler studies. Stroke 41: 2697-2704.

9. Reinhard M, Roth M, Guschlbauer B, Harloff A, Timmer J, et al. (2005) Dynamic cerebral autoregulation in acute ischemic stroke assessed from spontaneous blood pressure fluctuations. Stroke 36: 1684-1689.

10. Reinhard M, Wihler C, Roth M, Harloff A, Niesen WD, et al. (2008) Cerebral autoregulation dynamics in acute ischemic stroke after rtPA thrombolysis. Cerebrovasc Dis 26: 147-155.

11. Immink RV, van Montfrans GA, Stam J, Karemaker JM, Diamant M, et al. (2005) Dynamic cerebral autoregulation in acute lacunar and middle cerebral artery territory ischemic stroke. Stroke 36: 2595-2600.

12. Reinhard M, Rutsch S, Lambeck J, Wihler C, Czosnyka M, et al. (2012) Dynamic cerebral autoregulation associates with infarct size and outcome after ischemic stroke. Acta Neurol Scand 125: 156-162.

13. Boas DA, Dale AM, Franceschini MA (2004) Diffuse optical imaging of brain activation: approaches to optimizing image sensitivity, resolution, and accuracy. Neuroimage 23 Suppl 1: S275-288.

14. Huppert TJ, Hoge RD, Diamond SG, Franceschini MA, Boas DA (2006) A temporal comparison of BOLD, ASL, and NIRS hemodynamic responses to motor stimuli in adult humans. Neuroimage 29: 368-382.

15. Schroeter ML, Cutini S, Wahl MM, Scheid R, Yves von Cramon D (2007) Neurovascular coupling is impaired in cerebral microangiopathy--An event-related Stroop study. Neuroimage 34: 26-34.

16. Schytz HW, Ciftçi K, Akin A, Ashina M, Bolay H (2010) Intact neurovascular coupling during executive function in migraine without aura: interictal near-infrared spectroscopy study. Cephalalgia 30 : 457-466.

17. Tachtsidis I, Tisdall M, Delpy DT, Smith M, Elwell CE. 2008. Measurement of cerebral tissue oxygenation in young healthy volunteers during acetazolamide provocation: a transcranial Doppler and nearinfrared spectroscopy investigation. Adv Exp Med Biol 614: 389-396.

18. Reinhard M, Wehrle-Wieland E, Grabiak D, Roth M, Guschlbauer B, et al. (2006) Oscillatory cerebral hemodynamics--the macro- vs. microvascular level. J Neurol Sci 250: 103-109.

19. Phillip D, Schytz HW, Selb J, Payne S, Iversen HK, et al. (2012) Low frequency oscillations in cephalic vessels assessed by near infrared spectroscopy. Eur J Clin Invest 42: 1180-1188.

20. Muehlschlegel S, Selb J, Patel M, Diamond SG, Franceschini MA, et al. (2009) Feasibility of NIRS in the neurointensive care unit: a pilot study in stroke using physiological oscillations. Neurocrit Care 11: 288-295.

21. Cooper RJ, Selb J, Gagnon L, Phillip D, Schytz HW, et al. (2012) A systematic comparison of motion artifact correction techniques for functional near-infrared spectroscopy. Front Neurosci 6: 147.

22. Obrig H (2014) NIRS in clinical neurology - a 'promising' tool? Neuroimage 85 Pt 1: 535-546.

23. Schroeter ML, Schmiedel O, von Cramon DY (2004) Spontaneous lowfrequency oscillations decline in the aging brain. J Cereb Blood Flow Metab 24: 1183-1191.

24. Schroeter ML, Bücheler MM, Preul C, Scheid R, Schmiedel O, et al. (2005) Spontaneous slow hemodynamic oscillations are impaired in cerebral microangiopathy. J Cereb Blood Flow Metab 25: 1675-1684. 